\title{
Celulares al aula. Un estudio de caso en la Región Metropolitana
}

\author{
Luis Ramírez Bahamonde ${ }^{1}$
}

Recibido 11 de julio 2018 · Aceptado 19 de octubre 2018

\section{RESUMEN}

El siguiente artículo se desprende de la investigación realizada en 2016 en la comuna de Quilicura, titulada: "Celulares Smartphone en el aula y su impacto en el aprendizaje". Se trabajó en $6^{\circ}$ básico en la asignatura de Historia y Geografía.

Se podrá observar el tipo de investigación realizada, la forma en que se trataron los datos, los diversos participantes de esta investigación y las conclusiones obtenidas.

Palabras clave: Smartphone, Educación, Innovación, Trabajo colaborativo.

\section{Cellulars to the classroom. A case study in the Metropolitan Region}

\section{ABSTRACT}

The following article is derived from the research carried out in 2016 in the commune of Quilicura, entitled: "Smartphone phones in the classroom and their impact on learning". The levels in which they worked were 6th grade and the subject was History and Geography. It will be possible to observe the type of research carried out, the way in which the data were treated, the various participants of this research and the pertinent conclusions.

Keywords: Smartphone, Education, Innovation, Collaborative work

I Magíster en Educación de la UCSH. Encargado de la Unidad de Estudios Generales de la Universidad Academia de Humanismo Cristiano. luisarturoramirez@gmail.com 


\section{Introducción}

En pleno siglo XXI la tecnología está presente en cada momento de nuestros días y, desde hace algunos años, el ingreso de celulares de tipo Smartphone a nuestro mercado es cada vez más impactante. Un hecho significativo es que en nuestro país hay más Celulares Smartphone (CS) que habitantes: actualmente hay más de 23 millones de abonados en nuestro país (www.subtel.gob.cl), y no solo en cantidad se nota la importancia, sino que también en su impacto en la sociedad, donde se ha metaforizado el aparato como "una extensión de la mano", por el uso casi imprescindible que se le ha dado a este recurso tecnológico en nuestro quehacer.

En este punto, como docentes, nos preguntamos ¿qué tan importante es el uso actual de estos dispositivos en los colegios de nuestro país? ¿Son herramientas factibles de trabajo al interior del aula? ¿Cuál es su impacto real? ¿Qué tan dispuesta está la comunidad escolar para trabajar con estos dispositivos? ¿Existe un impacto en el rendimiento académico de los estudiantes al utilizar los celulares como recurso pedagógico?

Para intentar dar una respuesta significativa a estos cuestionamientos es que en 2016, en la escuela Weston Academy de la comuna de Quilicura, se realizó una investigación que contó con la colaboración de una profesora de Historia y Geografía que impartía clases en dos sextos básicos. Se realizó una intervención durante una unidad de aprendizaje, en los meses de abril y mayo de 2016, que consistió en aplicar en uno de los cursos una metodología colaborativa usando los celulares smartphone de los estudiantes y las aplicaciones Kaboot y Socrative, y en el curso paralelo continuar con el trabajo que regularmente lleva la profesora en sus clases. Al finalizar la intervención se analizó el rendimiento escolar en ambos grupos por medio de una evaluación de carácter sumativo. De forma complementaria, se recogió información de sujetos de la comunidad educativa, como profesores, apoderados, equipo directivo y estudiantes, que da cuenta de sus valoraciones y percepciones frente al uso de una metodología didáctica que trabaja con celulares smartphone. 


\section{Justificación}

En la actualidad, cada vez más niños y adolescentes tienen acceso a celulares smartphone y muchos apoderados defienden su uso, por parte de los menores, por motivos de seguridad y comunicación efectiva. Nuestra realidad como docentes es que debemos lidiar clase a clase con estos nuevos dispositivos electrónicos que han llegado para quedarse.

Nuestras clases desde hace décadas dejaron de ser 100\% expositivas y hemos pasado de escribir con tiza a hacerlo con plumones. La tecnología va llegando a nuestras vidas y también lo hace a nuestros trabajos. Ya no planificamos en papel, ahora todo lo hacemos desde nuestros computadores de escritorio y/o portátiles, y también los celulares y tabletas son parte de nuestro quehacer docente.

El incentivo al uso de las Tecnologías de Información y Comunicación (TIC) viene sucediendo desde hace varios gobiernos a través de capacitaciones, fondos y proyectos, pero aún no se logra delimitar correctamente hacia dónde vamos con el uso de las tecnologías al interior de la sala clases, ni qué tipo de tecnología ni quienes deben utilizarla: si solo los docentes para exponer sus clases o también los estudiantes para volverse partícipes de su educación.

Investigaciones acerca del impacto de las TIC en el rendimiento escolar hay bastantes y la preocupación, evidenciada en políticas de Estado, por incorporar las TIC en educación ya es un hecho. Pero es importante identificar si existen relaciones directas en el rendimiento escolar y el uso de celulares smartphone en el aula y estudiarlos como un recurso independiente de las conocidas TIC, ya que, aunque pertenecen a esta área, poseen propiedades que los diferencian claramente del resto. Si este hecho es una constante en diversos contextos educativos, ya que puede formar parte importante del proceso de enseñanza-aprendizaje, incorporar con sentido pedagógico estos recursos es un paso que hay que dar pronto, de lo contrario la incorporación de este recurso en el aula podría seguir siendo considerado como un elemento distractor. 


\section{Planteamiento del problema}

\section{Aprendizaje por medio de celulares smartphone}

Los CS son parte de los dispositivos móviles, tal como las tablets y los netbooks, entre otros. Es un recurso que ha tenido una relación cada vez más fuerte con la sociedad. A diferencia de otros recursos tecnológicos, los CS han tenido una repercusión distinta, mucho más fuerte, situación que los ha llevado a instalarse en varios aspectos de nuestra vida diaria, así también se ha estado situando en educación. A continuación se presenta la importancia de este dispositivo, primero describiendo una situación general en la sociedad, luego su relación con la educación, para finalizar con las características que presentan estos dispositivos em la vida de alumnos y profesores.

El uso de teléfonos celulares exhibe un crecimiento exponencial en los últimos IO años, con índices de suscriptores que representan el 99\% de la población mundial y superan el I00\% en muchos países (Unesco, 2012). Esto no significa que la cobertura sea universal, ya que algunas personas poseen más de un dispositivo mientras otros no tienen ninguno. De todas formas, esto indica que los niveles de adopción son extremadamente altos para todos los grupos socioeconómicos en Latinoamérica.

En América Latina la distribución de la tecnología y su infraestructura refleja las desigualdades en la educación. Chile está dentro de los países con mayores ingresos, así como Argentina, Paraguay, Panamá y Puerto Rico, entre otros. Coincide además con tener un nivel de infraestructura más avanzado. De manera transversal, el grado de penetración de celulares en América Latina es muy alto, incluso en los grupos socioeconómicos más bajos (Unesco, 2012), cifra que se refleja proporcionalmente en Chile. Estos dispositivos, entonces, son un gran candidato para programas de aprendizaje móvil, ya que la mayoría de la población ya posee este dispositivo. Por primera vez en la historia, la mayoría de las personas puede costear la adquisición personal de TIC en forma de dispositivos móviles, en particular CS. 
Uno de los momentos más complejos al momento de definir si utilizamos o no los CS como recurso pedagógico es delimitar su uso, ya que actualmente la industria relacionada con esta tecnología tiene más opciones de entretenimiento y diversión que opciones ligadas a la educación y/o investigación. Esto promueve que los encargados de tomar decisiones referentes a la política educativa del país o a la convivencia escolar de cada establecimiento educacional opten por prohibir su uso a los estudiantes durante las clases y, en algunos casos extremos, esta medida llega también a los docentes, ya que ven en estos aparatos elementos distractores $y /$ o nocivos para la educación. A pesar de esta realidad, poco a poco las tecnologías móviles y, por ende, los CS, han llegado a desempeñar un papel importante en las economías y en el conjunto de la sociedad. Los dispositivos móviles han incidido prácticamente en todos los campos, desde la banca hasta la política, y se emplean para acrecentar la productividad en numerosos sectores (Unesco, 2013). A medida que estos dispositivos ganan protagonismo en todo el mundo, va surgiendo interés por el "aprendizaje móvil”, entendido como una manera de apoyar al aprendizaje en un medio en el que diversos elementos, como la espontaneidad, la personalización, la informalidad, la contextualización, la portabilidad, la conveniencia, la adaptabilidad, la integración y la disponibilidad, juegan un papel relevante (Laouris, 2005). Sharples (2005) describe el aprendizaje como un proceso de acercamiento al conocimiento, en el que los participantes, en cooperación con sus compañeros y profesores, construyen en forma conjunta la interpretación de su mundo. Esta definición da a las tecnologías móviles un rol especial, porque incrementa las posibilidades de comunicación y conversación.

La capacidad de extender las experiencias educativas más allá de las aulas y posibilitar el aprendizaje no formal e informal, es un atributo clave del aprendizaje móvil, que encierra un enorme potencial para que el aprendizaje sea más personalizado y pertinente. Sin embargo, en este contexto, la movilidad "no denota sólo movilidad física, sino la oportunidad de superar restricciones físicas al tener acceso a las personas y a los recursos de aprendizaje digital independientemente del lugar y del tiempo" (Kukulska-Hulme, 2010). Así pues, el aprendizaje móvil puede perfectamente darse también en el aula. 


\section{Delimitación del problema}

La investigación buscó determinar el impacto del uso de dispositivos CS, como metodología de enseñanza-aprendizaje en el aula, en el rendimiento escolar.

Esta investigación se realizó en la escuela Weston Academy de la comuna de Quilicura.

Se analizó el rendimiento escolar y una nueva metodología de trabajo con base en el uso de CS en dos cursos de $6^{\circ}$ básico en sus respectivas aulas. El establecimiento corresponde al sistema privado de educación.

Ambos cursos trabajaron la misma unidad de aprendizaje en la asignatura de Historia y con la misma profesora. Un curso implementó el uso de CS en al menos dos horas de clases a la semana, y el otro curso mantuvo su metodología tradicional.

El tiempo en que se realizó esta investigación fue de siete semanas, lo que equivale a $2 \mathrm{I}$ horas cronológicas según el Ministerio de Educación.

Dentro de los recursos disponibles, estos corresponden a horas de trabajo; conexión a internet; computador, como recursos esenciales del investigador. A estos se suman los recursos en el aula, que son los CS de cada uno de los alumnos; conexión a internet en el aula; proyector y computador para el profesor, además de los propios de éste, como plumón y libros de la disciplina.

También, a los recursos de carácter tecnológico señalados, se sumaron las aplicaciones móviles (App) usadas por los estudiantes y la docente. En particular, se trabajó con las aplicaciones Kaboot y Socrative, porque ambas presentan funcionalidades en las que el docente, de forma previa a la clase, sube actividades de acuerdo con su planificación. Éstas se encuentran en modalidad de preguntas abiertas o de selección, y los estudiantes, en grupo o individualmente, responden desde sus equipos móviles. El interés de la investigación, además del uso de este tipo de recursos, se vincula con un trabajo docente que plantee una metodología idónea 
en favor de los aprendizajes. Para ello se capacitó en el uso de las App para CS y en la metodología.

\section{Objetivo General}

Determinar el impacto en el aprendizaje al utilizar como medio de enseñanza los equipos celulares smartphone en el aula en estudiantes de $6^{\circ}$ año básico de la escuela Weston Academy de Quilicura.

\section{Objetivos específicos}

I. Establecer la relación entre el rendimiento en estudiantes de acuerdo a las calificaciones obtenidas en el aprendizaje utilizando la intervención metodológica de CS en el aula, y el rendimiento de estudiantes que no utilizan este equipo móvil en su proceso didáctico.

2. Analizar las percepciones, valoraciones y conocimiento técnico del uso de CS en el proceso de enseñanza-aprendizaje de alumnos y profesores.

3. Analizar las percepciones y valoraciones de parte del equipo directivo, encargado computacional y apoderados, acerca del uso de CS en el proceso de enseñanza-aprendizaje.

\section{Variables}

La investigación, aunque presenta un carácter mixto, tiene una tendencia mayor a lo cuantitativo y, bajo ese aspecto, busca relaciones entre variables independientes (VI) y dependientes (VD). La VI corresponde especialmente a la metodología didáctica con CS o sin éste, y la variable dependiente (VD) del estudio se asocia con el rendimiento académico.

\section{Metodología de enseñanza-aprendizaje}

Actualmente existen diversos métodos de enseñanza-aprendizaje en el mundo. Para este artículo solo mencionaremos aquellas metodologías que en la investigación aparecen detalladas: 
- Aprendizaje colaborativo.

- Aprendizaje orientado a proyectos.

- Contrato de aprendizaje.

- Aprendizaje basado en problemas.

- Lección Magistral.

- Estudio de casos.

- Simulación y juegos.

En nuestro país y de forma tradicional, es la clase magistral la preferida por muchos colegios y docentes, pero la educación para el siglo XXI nos muestra la necesidad de permitir al estudiante ser un participante activo de su propio proceso de aprendizaje.

Actualmente, las diversas teorías de enseñanza-aprendizaje reconocen la gran importancia de las relaciones sociales para crear conocimiento; la relación directa/indirecta con un "otro" y saber trabajar en equipo son ejes fundamentales de la educación del siglo XXI.

El aprendizaje colaborativo tiene como tesis fundamental que las relaciones sociales determinan el desarrollo cognitivo y la creación de conocimiento, así como la mediación semiótica de los procesos cognitivos, es decir, el funcionamiento psicológico está mediado por instrumentos y signos. Podemos decir que en el aprendizaje colaborativo se produce la unión e intercambio de esfuerzos entre los integrantes que conforman un grupo; en el caso que nos ocupa, entre alumnos-alumnos, alumnos-profesores o profesores-profesores; de tal manera que el objetivo común y grupal que se persigue produzca, al final del proceso, un beneficio individual en todos y cada uno de los participantes. Entendemos que el aprendizaje colaborativo se sirve de estrategias cooperativas, entendiendo que cooperar significa trabajar juntos para alcanzar objetivos compartidos (García \& Hernández, 2012).

La colaboración y la cooperación son cada vez más utilizadas, no solo en el ámbito educativo, sino que también en los niveles económico, empresarial y político. Podemos ver cómo la metodología basada en proyectos y la colaboración es cada vez más utilizada en sistemas educativos como los de Finlandia y Alemania, aprovechando sus 
múltiples beneficios para el desarrollo de la autonomía del estudiante y permitiéndole construir su propio aprendizaje.

Sin duda, estamos frente a grandes cambios de nivel metodológico y social; en este sentido los colegios, institutos y universidades, deben ser susceptibles a estos cambios casi culturales, y deben dar respuesta y adaptarse a las nuevas necesidades de la sociedad.

\section{Marco metodológico}

\section{Enfoque de la investigación}

El enfoque fue mixto, partiendo por una fase basada en el enfoque cuantitativo y, posteriormente, recogiendo información cualitativa que complementara el modelo cuantitativo. Debido a las particularidades del estudio y de su ejecución, se presenta como un diagnóstico general que entregue un panorama de forma integral; por ello, fue menester abarcar desde el enfoque cuantitativo y cualitativo, dependiendo del sujeto implicado, rescatando las fortalezas que proporcionan ambos enfoques. De este modo, los análisis se plasmaron en integración y discusión conjunta de los datos provenientes de ambas fuentes.

La investigación otorgó un lugar predominante al enfoque cuantitativo, dados los objetivos que persigue y la hipótesis que pretende corroborar. Por tanto, el punto central fue abarcado mediante el análisis del rendimiento académico de los alumnos en la unidad evaluada y el análisis de las encuestas de percepción aplicadas, respondiendo en una primera etapa a un enfoque cuantitativo en un diseño experimental.

El enfoque mixto de esta investigación permitió apreciar los distintos ámbitos en el que se desarrolla la labor docente, es decir, el instrumento de medición cualitativo, desde ahora llamado "encuesta”, posibilitó conocer diferentes variables en torno a la investigación. Se aplicaron encuestas a los estudiantes, docentes y directivos, para conocer sus apreciaciones sobre el impacto del uso de CS como herramienta de aprendizaje durante el inicio, desarrollo y cierre de la investigación. Por otro lado, el porcentaje de aprobación y el rendimiento mostrado por cada grupo curso al finalizar la unidad de aprendizaje permitió el 
análisis comparativo y cuantitativo entre ambos cursos, y responder nuestro cuestionamiento inicial relacionado al impacto que puedan generar los CS en el desarrollo académico de nuestros estudiantes.

\section{Método de investigación y tipo de alcance del estudio.}

La investigación corresponde a un diseño explicativo, de carácter cuasiexperimental, el cual consiste en "un estudio en el que se manipulan intencionalmente una o más variables independientes (supuestas causas-antecedentes), para analizar las consecuencias que la manipulación tiene sobre una o más variables dependientes (supuestos efectos-consecuentes), dentro de una situación de control para el investigador” (Hernández \& Collado, 2003).

La investigación realizada no pudo controlar todas las variables presentes que se interrelacionan de una u otra manera al interior de la sala de clases en ambos cursos; no obstante, se pretendió "garantizar la explicación, interviniendo y creando las condiciones de investigación más adecuadas a los intereses del investigador, aunque todo ello suponga violentar las condiciones normales de producción del fenómeno" (Bisquerra, 2012).

El curso $6^{\circ}$ A corresponde a nuestro grupo experimental, pues son estos quienes tendrán acceso a las aplicaciones desde sus propios celulares (estímulo experimental), proceso que se alcanzó previa comunicación y autorización de sus apoderados.

Mientras que el curso $6^{\circ} \mathrm{B}$ corresponde al grupo de control, pues ellos continuarán con las clases sin intervención. No obstante, ambos cursos forman parte de la investigación.

\section{Criterio de selección de informantes: muestreo no probabilístico de carácter intencional}

Esta investigación fue desarrollada en la Escuela Básica Weston Academy, ubicada en Av. Bernardo O'Higgins OI20 de la comuna de Quilicura, de dependencia particular subvencionada. Posee énfasis en el desarrollo integral, la experiencia académica y deportiva, de orientación laica y con programas de formación en convivencia 
escolar, orientación, prevención de drogas y alcohol, educación de la sexualidad, cuidado del medio ambiente, promoción de la vida sana, actividades de acción social y actividades de pastoral. Los sujetos de estudio correspondieron a estudiantes de sexto año básico, correspondientes a dos cursos, $6^{\circ}$ A y $6^{\circ} \mathrm{B}$, de composición mixta y un número total de $5 \mathrm{I}$ estudiantes.

No existió selección previa del establecimiento educacional, sino que su participación fue contextual y por acceso, ya que el colegio contó con la disposición para realizar la investigación, considerándola como un aporte a la innovación educativa; los otros establecimientos consultados no estuvieron dispuestos a participar dentro de la muestra, por motivos confidenciales.

Para el trabajo con los estudiantes se solicitó la autorización mediante la firma de un consentimiento por parte de los apoderados, en el que se les informó los objetivos de la investigación y el alcance de la misma, recalcando que los datos obtenidos eran confidenciales y solo con fines del presente proyecto. Los formularios fueron firmados por el 100\% de los involucrados. Se contó con la voluntad de participación de Paula Suazo, profesora titulada de Pedagogía en Historia de la UCSH, quien imparte clases en ambos cursos (6A y 6B) y trabaja desde hace cinco años en el colegio.

La intervención se acotó a la primera unidad de aprendizaje, denominada "Democracia y Sociedad", cuya duración es de un mes y medio, según la planificación del establecimiento.

\section{Técnicas de recolección de datos}

Se construyó una encuesta de recolección de datos que solicitaba los datos de identificación del estudiante e informaba acerca de los objetivos de la investigación, con el fin de que el consentimiento fuera adecuadamente informado.

Las dimensiones que se consultaron fueron: - Distracción en el aula. - Motivación para el aprendizaje. - Conocimiento técnico (uso del teléfono celular). - Participación en clases. - Rendimiento escolar. - Trabajo colaborativo. 
Todas las anteriores dimensiones fueron medidas en escala de Likert, por considerar la forma más óptima de recoger una percepción acerca de lo consultado. La escala tiene 5 aseveraciones, en donde I se inclina a la opción "Muy en desacuerdo" y 5 se inclina a "Muy de acuerdo". La información obtenida fue digitada en el software estadístico SPSS $囚$, en su versión I.9, y posteriormente analizada correlacionando las variables mencionadas anteriormente.

Por otro lado, en la fase cualitativa se aplicó una encuesta abierta a los docentes y directores del establecimiento, construida a partir de las dimensiones arrojadas desde los objetivos de la investigación.

Se utilizaron técnicas de análisis de información cuantitativa y cualitativa, por lo que esta investigación se considera de carácter mixto, como habíamos mencionado, debido a que el objeto de estudio requiere de un amplio espectro de análisis.

\section{Resultados}

Del análisis de la información cualitativa podemos concluir que los diferentes integrantes de la comunidad educativa que participaron de la encuesta Percepciones generales del uso de Celulares Smartphones tienen diversas valoraciones y percepciones, tanto positivas como negativas, frente al uso de estos dispositivos en el aula.

Algunas percepciones de los encuestados incluían la preocupación por la responsabilidad sobre el equipo: ¿quiénes debían hacerse responsables ante una pérdida, robo o caída? Muchos coincidieron que el potenciar el uso de estos equipos en el aula, como estrategia de aprendizaje, solo generaría un mayor trabajo y responsabilidad por parte del profesor. Otros consideraron que el uso constante de estos equipos al interior de la sala de clases solo generaría problemas de comportamiento y concentración.

En otro sentido, profesores y algunos miembros del equipo directivo del establecimiento consideraron que potenciar el uso de estos equipos es parte de un proceso natural de "evolución" pedagógica: si antes considerábamos que solo el docente podía entregar conocimientos, y ahora creemos que el estudiante debe ser partícipe en la construcción 
y adquisición de su propio conocimiento, ¿por qué negarse a utilizar nuevas tecnologías e insumos que se puedan adaptar a las necesidades educativas del siglo XXI?

Claramente, el equipo de docentes y directivos del establecimiento se cuestionó la capacidad técnica con que cuenta el colegio para dar abasto con internet, o de si todos los estudiantes eran dueños de un equipo smartphone con la tecnología suficiente para instalar las aplicaciones necesarias y usar pedagógicamente el aparato.

Las opiniones respecto del uso de esta tecnología fueron variadas, muchas mostraban lo motivantes y cercanos que son estos dispositivos para los estudiantes, y que, a su vez, permitían desarrollar el aprendizaje integrativo y el apoyo interactivo al aprendizaje.

Opiniones variadas, positivas y negativas, es el resultado de la investigación cualitativa realizada tanto a apoderados, profesores y equipo directivo.

Del análisis cuantitativo se pudo desprender, en las distintas tablas de comparación entre ambos cursos, que no existieron variaciones significativas en el rendimiento académico.

En función de estos resultados se infiere que no habría diferencia significativa en el rendimiento académico obtenido en la unidad de aprendizaje Democracia y Participación Ciudadana, de $6^{\circ}$ básico en la escuela Weston Academy de la comuna de Quilicura. La unidad de aprendizaje tuvo una duración de siete semanas, lo que equivale a 2I horas pedagógicas. La unidad se realizó sin mayores contratiempos en los grupos de control y experimental durante el respectivo periodo planificado por la profesora.

De esta forma, la hipótesis de trabajo que dice, "La intervención metodológica por medio de celulares smartphone en el aula implica una mejora significativa en el rendimiento de los estudiantes, observado en las calificaciones obtenidas" no fue confirmada. 


\section{Conclusiones}

La tecnología llegó para quedarse en un país en el que, actualmente, se venden más celulares smartphone que computadores de escritorio o notebooks.

Las tecnologías de aprendizaje móvil también han evolucionado, con plataformas cada vez más fáciles de utilizar, cursos a distancia y especializaciones a un solo click a precios mucho más accesibles que hace diez años.

La tecnología nos conecta y nos permite conocer realidades diversas y distantes.

Esta investigación buscó mediar entre las tecnologías móviles y nuestro actual -y tradicional- sistema de educación. Diversos estudios han demostrado que la clase magistral es la metodología de enseñanza aprendizaje más utilizada en nuestro país, y que pocos profesionales de la educación se atreven a utilizar otras metodologías, en parte por desconocimiento o por la necesidad de mantener el statu quo.

El aprendizaje colaborativo como metodología y el uso de celulares smartphone en el aula como un recurso significativo son ejes importantes de este estudio, en el que finalmente se asocian con el rendimiento escolar (RE). Éste tiene mucha más historia en investigaciones $y$, a su vez, se identifica una cantidad de variables o factores compuestos de una forma compleja que se pueden atribuir positivamente a mejorar el rendimiento del estudiante.

Durante este estudio se analizaron diversas variables que incidieron de diferentes formas en los resultados. Una variable fue la estructural, que el establecimiento contara con señal de WiFi adecuada para dar conectividad a todos los celulares fue un factor determinante en la adecuada implementación del experimento, según indicó al finalizar la investigación la profesora a cargo de realizarlo. Otra variable importante es la referida al contexto familiar que, sin dudarlo, influye de manera significativa en el aprendizaje de los estudiantes. El aspecto cognitivo que se observa desde los resultados cualitativos está focalizado respecto de la dinámica, entretención, aprendizaje interactivo por medio de los celulares, lo cual explica una mejor 
atención a las clases, mejor atención al aprendizaje y, por ende, incide en mejorar el proceso cognitivo de los estudiantes, general e individualmente. Sin embargo, a partir de los datos cualitativos, no se puede asegurar con certeza en qué niveles existe una mejoría en el rendimiento escolar; mientras que, en relación a lo cuantitativo, se rechazó la hipótesis, ya que los resultados obtenidos en la evaluación final de la unidad de aprendizaje no demostraron cambios significativos en el rendimiento académico.

La percepción general acerca del uso de los celulares smartphone es que se trata de herramientas útiles para la educación actual, pero los docentes encuestados no demostraron interés en cambiar de metodología de enseñanza, prefiriendo la clase magistral por sobre la metodología de aprendizaje colaborativo.

Es pertinente volver sobre este tema, debido a que la implementación del experimento no solo contemplaba la incorporación de los CS como herramientas pedagógicas, sino que, además, el cambio desde una metodología de clase magistral a una colaborativa, porque en variados estudios se ha demostrado que es esta metodología la que más se ve beneficiada con el uso constante de estos aparatos; en cambio, los docentes que participaron de esta investigación solo se mostraron interesados en incluir el uso de esta herramienta en sus actuales planificaciones y metodologías de trabajo, no en capacitarse y comenzar a utilizar la metodología de aprendizaje colaborativo.

Para finalizar, es menester plantear la fuerte necesidad de invertir tiempo y recursos en capacitación de nuestros docentes, comenzar a considerar un cambio metodológico en el proceso educativo e integrar con más fuerza las diversas tecnologías que nos ofrece el mercado: por qué no hacerlo con los CS, que presentan características como familiaridad, confianza y cercanía con el estudiante. El sistema educacional chileno debe modernizarse, tanto en la metodología de enseñanza-aprendizaje que utiliza como con las herramientas didácticas para desarrollar el proceso enseñanza-aprendizaje. Se considera significativo generar innovación y diversos cambios en el sistema de enseñanza, pues se comprende que el proceso tradicional de aprendizaje ya no funciona del todo bien. 
El presente estudio pretende además ser un aporte para investigaciones e intervenciones en el área, en referencia a metodologías de enseñanza y el uso de recursos móviles en el aula. Investigaciones ya citadas señalan que la metodología de aprendizaje colaborativo es la más efectiva con las TIC. También, estos mismos estudios señalan que la metodología de clase magistral es la que predomina en las aulas, por lo que sería conveniente aislar las variables en estudios en los que se determine su impacto en el aprendizaje.

\section{Referencias}

Bisquerra, R. (2012). Metodología de la investigación educativa. Madrid: La Muralla.

García, A. y Hernández, A. y. (20I2). La metodología del aprendizaje colaborativo a través de las TIC: una aproximación a las opiniones de profesores y alumnos. Revista Complutense de Educación, 23(I), I6I-I88.

Hernández, R. y Collado, C. (2003). Metodología de la Investigación. México D.F.: McGrawHill Interamericana.

Kukulska-Hulme, A. (2010). Learning cultures on the move: where are we heading? Journal of Educational Technology and Society, 13(4), 4-I4.

Laouris, V. (2005). We need an educationally relevant definition of mobile learning. En Proceedings of the 4th World Conference on Mobile Learning (pp. 290-294).

Sharples, M. y Taylor, J. (2005). Towards a theory of mobile learning. telecomunicaciones, S. d. (I4 de 9 de 2016). Subtel. Recuperado de www.subtel.gob.cl

Unesco. (2012). Aprendizaje móvil para docentes temas globales. Recuperado de http:/ / unesdoc.unesco.org/images/002I/002164/2I6452s.pdf.

Unesco. (2013). Aprendizaje móvily políticas cuestiones clave. Recuperado de http:// unesdoc.unesco.org/images/0021/002176/217638s.pdf. 